\title{
Retrospective study of a series of 850 patients with temporomandibular dysfunction (TMD). Clinical and radiological findings
}

\author{
Rafael Poveda-Roda ${ }^{1}$, José V. Bagán ${ }^{2}$, Yolanda Jiménez-Soriano ${ }^{3}$, Antonio Fons-Font ${ }^{4}$ \\ ${ }^{1} \mathrm{MD}$ PhD. Assistant in Stomatology. Departament of Stomatology. General Universitary Hospital of Valencia \\ ${ }^{2}$ MD PhD. Professor and Chairman. Departament of Stomatology. General Universitary Hospital of Valencia \\ ${ }^{3} \mathrm{MD}$ PhD. Professor of Oral Medicine. University of Valencia \\ ${ }^{4} \mathrm{MD} \mathrm{PhD}$. Professor of Prothesis and Oclusion. University of Valencia
}

Correspondence:

Hospital General Universitario

Servicio de Estomatología

Avda. Tres Cruces s/n

46014 - Valencia (Spain)

poveda@uv.es

Received: 22/02/2009

Accepted: 08/06/2009

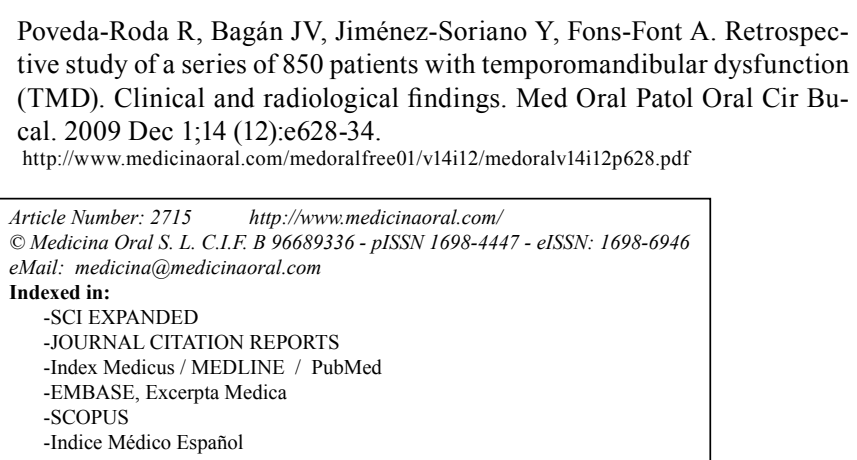

\begin{abstract}
Objective: To define the clinical and radiological characteristics of the four most common presentations of temporomandibular dysfunction-myofascial pain (MFP), disc displacement with reduction (DDWR), disc displacement without reduction (DDWoR), and osteoarthrosis (OR)-and to identify the differences among them.

Material and methods: The study comprised a series of 850 patients (121 males and 729 females) seen between May 2003 and December 2006 in Valencia University General Hospital (Spain) for temporomandibular joint disease (TMJD). An analysis was made of the possible etiological factors (stress, traumatisms, sleep disturbances, parafunctional habits, reason for consultation), possible pain sensations in response to palpation of the masticatory muscles, joint sounds, etc. A panoramic X-ray study was made on a routine basis, and in some patients $(\mathrm{n}=54)$ the study was completed with a magnetic resonance imaging scan of the temporomandibular joints and related tissues. The differences between qualitative variables were examined by means of the chi-square test with $\mathrm{R} \times \mathrm{C}$ contingency tables and the Z-test, while quantitative variables were contrasted by analysis of variance (ANOVA) and post hoc testing (Scheffe).

Results: The variables showing statistically significant differences among the four diagnostic categories were: patient age, sleep disturbances, stress, parafunctional habits, nibbling on hard objects and "parafunctions", reason for consultation, mandibular movements, "non-evaluable" molar and canine relationship, ligament hyperlaxity, and panoramic X-ray alterations.
\end{abstract}

Key words: Temporomandibular dysfunction, myofascial pain, disc displacement, osteoarthrosis, pain, TMD. 


\section{Introduction}

Many disease processes are able to affect the temporomandibular joint (TMJ), though four disorders either alone or in combination account for the immense majority of cases requiring medical attention: myofascial pain (MFP), disc displacement with reduction (DDWR), disc displacement without reduction (DDWoR), and osteoarthrosis (OR).

Classically, one of the most important deficiencies in the study of temporomandibular joint disease (TMJD) has been the lack of valid, reliable and reproducible criteria for the precise classification of the disorders.

The models proposed by Truelove et al. (Clinical Diagnostic Criteria for Temporomandibular Disorders, CDC/ TMD) (1) and by Dworkin et al. (Research Diagnostic Criteria for Temporomandibular Disorders, RDC/TMD) (2) are useful tools for the clinician, and are effective for conducting epidemiological studies of the most common disease processes affecting the TMJ.

The descriptive studies of TMJD have focused either on the identification of signs and symptoms in the general population (regardless of whether such manifestations are perceived or not by the patient), or on classification of the signs and symptoms in patients seen for TMJ problems (and thus interpreted as constituting an expression of disease). Few series have described the clinical characteristics (including the signs registered by imaging techniques) of patients structured according to the four most common forms of TMJD commented above (3-5).

The present study aims to define the differential clinical and epidemiological parameters of the four most prevalent presentations of TMJ pathology (MFP, DDWR, DDWoR and OA) in a series of 850 patients seen in the Service of Stomatology of Valencia University General Hospital (Valencia, Spain).

\section{Material and Methods}

A retrospective study was made of a series of 881 patients referred to the Service of Stomatology of Valencia University General Hospital (Spain) between May 2003 and December 2006. Thirty-one patients were excluded due to incomplete registry of the clinical data. The final study sample therefore totaled 850 patients.

All patients were informed of the use of their disease information for the conduction of this study, and informed consent was obtained in all cases.

We recorded the antecedents of trauma in the orofacial region. Sleeping difficulties, premature loss of sleep, or several awakenings at night were regarded as sleep disturbances. The patients were questioned about stress, and the reply (positive or negative) was registered in the case history. As regards parafunctional habits, the patients were questioned about tooth clenching, nibbling on pencils or pens, nail biting or frequent chewing of gum. Each of these parameters were recorded individually. Any other reported type of parafunction was registered as "other parafunctional habits".

Muscle palpation included the temporal, masseter, internal pterygoid, external pterygoid, digastric and sternocleidomastoid muscles. Palpation was carried out according to the recommendations of Dworkin et al. as regards the applied pressure (approximately $1 \mathrm{~kg}$ for extraoral palpation and about $0.5 \mathrm{~kg}$ for intraoral exploration) (2).

A dry and brief sound upon mandibular aperture or closure was recorded as a click (opening or closing click, depending on when the sound was produced). A sustained metal friction against sand-like sound during opening or closing of the mouth was registered as coarse crepitus.

Measurements were made of the mandibular movements corresponding to maximum interincisal aperture and to left and right lateralization. End feel evaluation (forced passive aperture over maximum active aperture) was limited to quality (hardness and pain).

The Beighton test measures systemic ligament laxity by evaluating 9 joints (metacarpophalangeal joint of the thumb and little finger of both hands, both elbows, both knees, and the lumbosacral joint). Values over 4 express variable degree of ligament laxity. We registered the test score with the definition of two groups: $\leq 4$ or $>4$. All the explorations were made by the same investigator (YJS).

In the present study, panoramic X-rays were used to explore the condylar morphology, recording the presence of condylar flattening and osteophytes. The evaluation was made by a single examiner blinded to the patient history and results of the clinical evaluation.

A principal diagnosis was established in each case, based on the following criteria:

Myofascial pain (MFP): Patients with spontaneous pain or pain in response to mastication, in any of the zones corresponding to the masticatory muscles. Positive muscle palpation findings at any of the above mentioned muscle points. Absence of joint sounds that prove reproducible in a stable manner.

Disc displacement with reduction (DDWR): Patients with a history of joint sounds. Detection of opening or of opening and closing clicks. The condition can be associated to pain in the lateral or posterior pole of the TMJ, in response to palpation. In this clinical context, the diagnosis of DDWR prevails even when painful muscle points are detected. The presence of MRI findings compatible with DDWR is considered to be diagnostic.

Disc displacement without reduction (DDWoR): Limitation of maximum interincisal aperture to under 35 $\mathrm{mm}$, supported by a prior history of joint sounds that are not noted at the time of the exploration. Other indicative 
signs are a hard end feel and deflections or deviations of the mandible in opening maneuvers. The presence of MRI findings compatible with DDWoR is considered to be diagnostic.

Osteoarthrosis (OA): This disorder is characterized by a history of pain in both TMJs, associated to morning stiffness lasting at least 30 minutes. Coarse crepitus auscultation. The presence of osteophytes or of condylar flattening in this clinical context is considered to be diagnostic of osteoarthrosis.

The data were analyzed using the Statistical Package for Social Sciences (SPSS) version 13.0. The qualitative variables were studied using the chi-square test with $\mathrm{R} \times \mathrm{C}$ contingency tables and the two-tailed Z-test in those cases where the differences proved statistically significant $(\mathrm{p}<0.05)$

Quantitative variables were contrasted by analysis of variance (ANOVA) and post hoc testing (Scheffe test) in those cases where the differences proved statistically significant $(\mathrm{p}<0.05)$. In application to the MRI findings, we determined the sensitivity, specificity, positive predictive value (PPV) and negative predictive value (NPV) of the clinical diagnosis, using the imaging technique as reference standard.

\section{Results}

The most relevant results corresponding to the series of 850 patients are reported in Table 1 .

The mean age was 40.5 years $(\mathrm{SD} \pm 18.7)$ (range $13-85$ years). The male / female ratio was $1 / 6.2$, and proved remarkably stable for each of the main diagnostic groups. The diagnostic distribution in our series was as follows: myofascial pain (35.2\%), disc displacement with reduction (44.8\%), disc displacement without reduction (6.5\%), and osteoarthrosis (13.4\%).

Comparison of the diagnostic groups in relation to sleep disturbances revealed $\chi^{2}=32.93 ; p<0.05$ - significant differences being detected between MFP (51.4\%) and DDWR (31.2\%), and OA (52.4\%) and DDWR. In the case of subjective permanent stress sensation, the differences found were between MFP (62.8\%) and DDWR (49.9\%).

Parafunctional habits were recorded in $63.2 \%$ of the study sample. The most common such disorder was teeth clenching (in $41.2 \%$ of cases). The habit of chewing gum was significantly more frequent in cases of disc displacement $(18.2 \%$ and $21.1 \%$, respectively) than in OA $(8.3 \%)(\mathrm{p}<0.05)$; and nibbling on hard objects (pencils and ballpoint pens) was significantly more common

Table 1. Reasons for consultation.

\begin{tabular}{|c|c|c|c|c|c|}
\hline & MFP $^{l}$ & DDWR $^{2}$ & DDWoR $^{3}$ & $\mathbf{O A}^{4}$ & Observations \\
\hline Frequency & $35.2 \%$ & $44.8 \%$ & $6.5 \%$ & $13.4 \%$ & $\mathrm{~N}=850$ patients \\
\hline$\delta / 9$ & $14.7 \% / 85.3 \%$ & $14.2 \% / 85.8 \%$ & $12.7 \% / 87.3 \%$ & $14 \% / 86 \%$ & $\begin{array}{c}\text { Global } \\
14.2 \% / 85.8 \%\end{array}$ \\
\hline Age (mean in years) & 41.9 & 31.1 & 31.0 & 56.0 & $F=90.95 p<0.05$ \\
\hline $\begin{array}{l}\text { History } \\
\text { Sleep disturbances } \\
\text { Stress }\end{array}$ & $\begin{array}{l}51.4 \% \\
62.8 \% \\
\end{array}$ & $\begin{array}{l}31.2 \% \\
49.9 \% \\
\end{array}$ & $\begin{array}{c}35.2 \% \\
50 \%\end{array}$ & $\begin{array}{l}52.4 \% \\
65.1 \% \\
\end{array}$ & $\begin{array}{l}\mathrm{X}^{2}=32.93 \mathrm{p}<0.05 \\
\mathrm{X}^{2}=11.53 \mathrm{p}<0.05\end{array}$ \\
\hline $\begin{array}{l}\text { Parafunctions } \\
\text { Chewing gum } \\
\text { Nibbling on hard } \\
\text { objects }\end{array}$ & $\begin{array}{l}13.3 \% \\
2.9 \%\end{array}$ & $\begin{array}{l}18.2 \% \\
8.2 \%\end{array}$ & $\begin{array}{c}21.1 \% \\
7.7 \%\end{array}$ & $\begin{array}{l}8.3 \% \\
1.9 \%\end{array}$ & $\begin{array}{l}\mathrm{X}^{2}=9.27 \mathrm{p}<0.05 \\
\mathrm{X}^{2}=11.17 \mathrm{p}<0.05\end{array}$ \\
\hline Joint sounds & $18.7 \%$ & $94.0 \%$ & $27.3 \%$ & $88.5 \%$ & $\begin{array}{c}\mathrm{X}^{2}=446.11 \\
\mathrm{p}<0.05\end{array}$ \\
\hline $\begin{array}{l}\text { End feel } \\
\text { Soft and painful } \\
\text { Hard and painless } \\
\text { Hard and painful }\end{array}$ & $\begin{array}{l}71.3 \% \\
1.0 \% \\
7.5 \%\end{array}$ & $\begin{array}{c}56.5 \% \\
2.4 \% \\
3.2 \%\end{array}$ & $\begin{array}{c}56.4 \% \\
9.1 \% \\
14.5 \%\end{array}$ & $\begin{array}{c}57.1 \% \\
6.3 \% \\
4.5 \%\end{array}$ & $\mathrm{X}^{2}=56.17 \mathrm{p}<0.05$ \\
\hline $\begin{array}{l}\text { Non-evaluable } \\
\text { Angle relationship }\end{array}$ & $26.8 \%$ & $16.1 \%$ & $15.2 \%$ & $38.8 \%$ & $\mathrm{X}^{2}=30.22 \mathrm{p}<0.05$ \\
\hline Beighton test $>4$ & $16.1 \%$ & $36.4 \%$ & $27.3 \%$ & $10.5 \%$ & $\mathrm{X}^{2}=51.81 \mathrm{p}<0.05$ \\
\hline $\begin{array}{l}\text { Panoramic X-ray } \\
\text { alterations }\end{array}$ & $4.0 \%$ & $6.0 \%$ & $16.4 \%$ & $35.6 \%$ & $\mathrm{X}^{2}=83.3 \mathrm{p}<0.05$ \\
\hline
\end{tabular}

1 Myofascial pain; 2 Disc displacement with reduction; 3 Disc displacement without reduction; 4 Osteoarthrosis 
Table 2. Reasons for consultation.

\begin{tabular}{|l|c|c|c|c|c|c|c|c|}
\hline & \multicolumn{2}{|c|}{ Myofascial pain } & \multicolumn{2}{|c|}{ DDWR } & \multicolumn{2}{c|}{ DDWoR } & \multicolumn{2}{c|}{ Osteoarthrosis } \\
\hline Reason consultation & $\mathrm{N}$ & $\%$ & $\mathrm{~N}$ & $\%$ & $\mathrm{~N}$ & $\%$ & $\mathrm{~N}$ & $\%$ \\
\hline Sounds & 10 & $3.3 \%$ & 85 & $22.3 \%$ & 1 & $1.8 \%$ & 7 & $6.1 \%$ \\
\hline Pain & 193 & $64.5 \%$ & 118 & $30.9 \%$ & 19 & $34.5 \%$ & 70 & $61.4 \%$ \\
\hline Block & 16 & $5.4 \%$ & 38 & $9.9 \%$ & 16 & $29.1 \%$ & 3 & $2.6 \%$ \\
\hline Sounds and pain & 27 & $9.0 \%$ & 62 & $16.2 \%$ & 5 & $9.1 \%$ & 16 & $14.0 \%$ \\
\hline Block and pain & 20 & $6.7 \%$ & 20 & $5.2 \%$ & 6 & $10.9 \%$ & 6 & $5.3 \%$ \\
\hline Sounds and block & 4 & $1.3 \%$ & 20 & $5.2 \%$ & 1 & $1.8 \%$ & 2 & $1.8 \%$ \\
\hline Sounds, block and pain & 29 & $9.7 \%$ & 39 & $10.2 \%$ & 7 & $12.7 \%$ & 10 & $8.8 \%$ \\
\hline
\end{tabular}

Table 3. Mandibular movements (in $\mathrm{mm}$ ).

\begin{tabular}{|l|l|l|l|l|l|c|c|c|}
\hline \multicolumn{2}{|c|}{} & Mean & SD* & SEM & $95 \%$ CI** & Median & Mode & Range \\
\hline Maximum aperture & MFP & 37.87 & 8.49 & 0.49 & $36.9-38.8$ & & & \\
& DDWR & 40.27 & 9.14 & 0.47 & $39.4-41.2$ & & & \\
& DDWoR & 29.04 & 8.67 & 1.17 & $26.7-31.4$ & & & \\
& OA & 36.95 & 7.76 & 0.73 & $35.5-38.4$ & & & \\
& Total & 38.25 & 9.12 & 0.31 & $37.6-38.7$ & 40 & 40 & $0-65$ \\
\hline Right lateralization & MFP & 5.9 & 3.05 & 0.18 & $5.6-6.3$ & & & \\
& DDWR & 6.52 & 2.82 & 0.14 & $6.2-6.8$ & & & \\
& DDWoR & 6.16 & 2.66 & 0.36 & $5.5-6.9$ & & & \\
& OA & 5.84 & 4.36 & 0.41 & $5.0-6.6$ & & & \\
& Total & 6.19 & 3.15 & 0.11 & $2.9-3.4$ & 6 & 5 & $0-20$ \\
\hline \multirow{2}{*}{ Left lateralization } & MFP & 6.19 & 3.17 & 0.18 & $5.8-6.6$ & & & \\
& DDWR & 6.89 & 2.82 & 0.14 & $6.6-7.2$ & & & \\
& DDWoR & 6.47 & 2.69 & 0.36 & $5.7-7.2$ & & & \\
& OA & 5.93 & 3.40 & 0.32 & $5.3-6.6$ & & \multirow{2}{*}{5} & $0-20$ \\
\hline
\end{tabular}

*SD: standard deviation; **CI: confidence interval

in DDWR $(8.2 \%)$ than in MFP $(2.9 \%)$ or in OA $(1.9 \%)$. The same was observed for "other parafunctional habits" (22.3\% in DDWR, $14.7 \%$ in MFP and $11.5 \%$ in OA).

The patient reasons for consultation are shown in Table 2. On considering pain, sounds and mandibular block both isolatedly and in combination, pain was seen to be present in $76.1 \%$ of the patients, joint sounds in $38.2 \%$, and joint block in $24.7 \%$. Joint sounds were detected at clinical exploration by palpation and auscultation in $61.3 \%$ of the patients. Such sounds were significantly more frequent in DDWR $(94.0 \%)$ than in the rest of diagnostic groups, and were also significantly more common in OA $(88.5 \%)$ than in MFP $(18, .7 \%)$ or DDWoR $(27.3 \%)\left(\chi^{2}=446,11 ; \mathrm{p}<0.05\right)$. Crepitants were recorded in $14.8 \%$ of the patients. In turn, joint clicks were registered in $52.5 \%$ of the subjects.

Surprisingly, muscle palpation proved positive at some muscle point in a full 844 of the 850 patients $(99.5 \%)$.
The results obtained in terms of mandibular movements are summarized in Table 3 .

A soft and painless end feel was more frequent $(\mathrm{p}<0.05)$ in DDWR (38.0\%) than in MFP (20.1\%). A soft and painful end feel was likewise more frequent in MFP $(71.3 \%)$ than in DDWoR $(56.4 \%)$ or OA $(57.1 \%)$. A hard and painless end feel proved more common in DDWoR (9.1\%) and OA (6.3\%) than in MFP (1.0\%), while a hard and painful end feel was more frequent in DDWoR (14.5\%) than in DDWR (3.2\%).

In $20.9 \%$ of the patients the Angle molar relationship could not be established due to the absence of reference teeth. The same occurred in $8.4 \%$ of the patients in the case of the canine Angle class. The patients with MFP showed a larger proportion $(\mathrm{p}<0.05)$ of "non-evaluable Angle molar relationships" $(26.8 \%)$ than the patients diagnosed with DDWR (16.1\%). The patients with OA likewise presented a greater proportion $(38.8 \%)(\mathrm{p}<0.05)$ than the patients with DDWR or DDWoR (16.1\% and 15.2\%, respectively). 
In the qualitative Beighton test, $25.1 \%$ of the study series showed a score of 4 or more, corresponding to a greater or lesser degree of ligament hyperlaxity. Statistically significant differences $(p<0.05)$ were found between myofascial pain and DDWR, between DDWR and OA, and between DDWoR and OA. The differences among diagnoses are shown in Tablel.

Condylar alterations were seen in $8.2 \%$ of the panoramic X-rays. Uni- or bilateral condyle flattening was recorded in $6.1 \%$ of cases, and uni- or bilateral osteophytes in $0.8 \%$. Both disorders concomitantly were observed in $1.3 \%$ of the cases.

MRI studies were made on the basis of the above specified criteria in 54 patients $(6.4 \%)$. The levels of sensitivity (S), specificity (Sp), positive predictive value (PPV) and negative predictive value (NPV) were established for the clinical criteria, using MRI as reference standard. Values were as follows: for DDWR S 0.8, Sp 0.71, PPV 0.40 and NPV 0.94; for DDWoR S 0.85, Sp 0.81, PPV 0.84 and NPV 0.90; for OA (condylar flattening) $\mathrm{S}$ 0.6, Sp 0.87, PPV 0.33 and NPV 0.95 and for OA (osteophytes) S 1, Sp 0.90, PPV 0.44 and NPV 1. Emphasis is placed on the need to interpret the results with caution, since MRI was performed only in certain clinical situations.

\section{Discussion}

With the exception of some sporadic series, most studies find TMJD to be more common in women than in men, with male/female ratios of between $1: 1.75$ and $1: 3$ $(6,7)$, in coincidence with our own observation (1:6.2). This same gender distribution is notoriously constant in the four different diseases categories considered (female prevalences of $85.3 \%$ in relation to MFP, $87.3 \%$ in DDWR, $87.3 \%$ in DDWoR, and $86.0 \%$ in the case of $\mathrm{OA})$. The studies made to explain the differences between males and females, evaluating psychosocial, endocrine, constitutional and behavioral factors, etc., have been unable to draw firm conclusions.

Isacsson et al. found that two-thirds of the patients requiring treatment for masticatory signs and symptoms presented disc displacement (3) - this figure being clearly greater than our own $(51.3 \%$, distributed as $44.8 \%$ DDWR and 6.5\% DDWoR). Mejersjo et al. in turn established a diagnosis of OA in $11.8 \%$ of their patients (4) - this figure being similar to our own (13.4\%). In the case of MFP, Huang et al. diagnosed the condition in $35.4 \%$ of their patients (5), though in their series 157 cases were diagnosed as corresponding to myofascial pain with arthralgia - in accordance with the RDC/ TMD (2).

Lindroth et al. found that patients with intracapsular pain (ICP) had better sleep quality than those with masticatory pain (6); our own observations coincide with these results. On evaluating sleep disturbances, it is intuitively accepted that the term includes difficulty falling asleep, easy awakening, or failure to sleep the required number of hours. In our study we have adhered to these same criteria. However, Collesano et al. studied excessive drowsiness in relation to TMJD, and reported this problem in $19 \%$ of their patients. On the other hand, drowsiness was more common in the group diagnosed with MFP (8).

In our series we evaluated subjective patient perception of stress, without applying specific tests. In our population we found that $62 \%$ of the patients with MFP, $49 \%$ of those with DDWR, $50.0 \%$ of those with DDWoR and $61.1 \%$ of the subjects with OA claimed to suffer stress, significant differences being recorded between MFP and DDWR. Kuttila et al., using the Symptoms of Stress Inventory (SOSI), reported significantly higher stress levels in the MFP group than in the patients with an arthrogenic-myogenic diagnosis or the group with only an arthrogenic diagnosis (9). Galdón et al. likewise recorded greater general distress levels in patients with MFP, particularly in relation to the somatization and anxiety domains, using the BSI-18 (10). Sieber et al., in a group of 413 adolescents, found stress to be significantly correlated to the number of painful muscle points (11). These data thus seem to support the hypothesis of stress as a cause of sustained muscle spasm and, ultimately, of myofascial pain.

Miyake et al. found teeth clenching to increase the risk of joint sounds, TMJ pain and limitations in mandibular movement (odds ratio, $\mathrm{OR}=1.86,1.78$ and 1.88 , respectively), in the general population (12). Huang et al. reported that patients with MFP and those with MFP plus joint pain were related to a significantly increased risk of teeth clenching (OR: 4.8 and 3.3, respectively) (5). In our study, teeth clenching yielded no significant differences among the different TMJD diagnostic groups. We only recorded those patients reporting tooth clenching, and did not include those subjects with wear facets but who were unaware of their habit. This could explain the differences seen with respect to the results of other authors.

Joint sounds traditionally have been regarded as a sign of TMJD. Coarse crepitus in turn has been associated with osteoarthrosis, and clicks (both on opening and on closing) with DDWR. However, Elfving et al. recorded some type of joint sound in $36 \%$ of their controls (13); similar data had already been published by Gross et al. in 1986 - with the documenting of joint sounds in $37 \%$ of the asymptomatic population (14).

In our study, both the global mean maximum aperture and the mean value corresponding to each of the diagnostic groups were clearly smaller than the threshold considered by Hirsch et al. to mark the normal limit: "The upper limit of the lowest decentile is $43 \mathrm{~mm}$, and any lesser maximum aperture should be regarded 
as a limitation of the latter"(15). Unlike in our series, Masumi et al. found no significant differences in terms of maximum aperture in the four groups of TMJD considered in their study (arthromyalgia, arthromyalgia with disc-condyle incoordination, isolated disc-condyle incoordination, and osteoarthrosis) - though the sample size involved $(n=41)$ could explain the absence of differences (16).

In the case of lateralization movements, our results (mean $=6.2 \mathrm{~mm}$ for right lateralization and $6.5 \mathrm{~mm}$ for left lateralization) are clearly below the value considered by Hirsch et al. (15) to represent the lower limit of normality $(8 \mathrm{~mm})$. In 1921, Wertheim observed that most individuals are able to move the mandible more towards the left side than to the right side. We likewise observed this difference in our series, though statistical significance was not reached.

In our study, in relation to both the angle molar relationship and canine relationship, the number of patients with MFP and non-evaluable occlusion was significantly greater than the number of patients with DDWR and non-evaluable occlusion. The number was likewise significantly greater in the OA group than in the two disc displacement groups. In line with our own study, Pullinger and Seligman found a loss of 5 or more posterior sector teeth to yield $\mathrm{OR}=2$ in terms of the capacity to predict OA of the TMJ (17). In this way, there appears to be a certain relationship between the loss of occlusal support and certain TMJ disorders, particularly myofascial pain - though on the basis of the results obtained it is not possible to postulate a causal relationship.

A little over a quarter of our patients (25.1\%) yielded a Beighton test score of 4 or higher. The DDWR group showed the highest percentage (36:4\%), while the lowest proportion corresponded to OA (10.5\%). Kavuncu et al. found ligament laxity to be more common in patients with TMJD than in the controls (18). Elfving et al. obtained results in coincidence with our own as regards DDWR, with ligament hyperlaxity in $39 \%$ of the patients (13).

Panoramic X-rays are of limited usefulness in application to TMJD, though their routine use is recommended. Magnusson et al. found radiological alterations in $25 \%$ of patients with TMJD. In $11 \%$ of the cases they recorded alterations that were unrelated to the TMJ, but that nevertheless required treatment (19). In our study we found alterations in $8.2 \%$ of the patients. In the group diagnosed with osteoarthrosis, alterations were detected in $35.6 \%$. The patients with symptoms and signs of OA but without radiological manifestations $(64.4 \%$ in our study) are an important confounding element - in the same way as the high percentage of healthy subjects with radiological evidence of OA. Crow et al. documented osteophytic changes in $42 \%$ of their subjects without TMJD, and evident osteophytes in 7\%. The same authors observed mild condyle flattening in $74 \%$ of the subjects without TMJD, and severe flattening in $15 \%$ (20). Adaptive bone remodeling can induce changes similar to those of osteoarthrosis, and this could explain the high prevalence of radiological alterations in healthy individuals.

In conclusion, certain variables of patient anamnesis and clinical exploration, such as sleep disturbances, stress, parafunctional habits, joint sounds, end feel, loss of occlusal support and ligament hyperlaxity may indicate differences among the four main diagnostic categories of TMJ disorders - though further studies are needed to assess the true importance of these variables in diagnosing the mentioned disease processes.

\section{References}

1. Truelove EL, Sommers EE, LeResche L, Dworkin SF, Von Korff M. Clinical diagnostic criteria for TMD. New classification permits multiple diagnoses. J Am Dent Assoc. 1992;123:47-54.

2. Dworkin SF, LeResche L. Research diagnostic criteria for temporomandibular disorders: review, criteria, examinations and specifications, critique. J Craniomandib Disord. 1992;6:301-55.

3. Isacsson G, Linde C, Isberg A. Subjective symptoms in patients with temporomandibular joint disk displacement versus patients with myogenic craniomandibular disorders. J Prosthet Dent. 1989;61:70-7.

4. Mejersjö C, Hollender L.TMJ pain and dysfunction: relation between clinical and radiographic findings in the short and long-term. Scand J Dent Res. 1984;92:241-8.

5. Huang GJ, LeResche L, Critchlow CW, Martin MD, Drangsholt MT. Risk factors for diagnostic subgroups of painful temporomandibular disorders (TMD). J Dent Res. 2002;81:284-8.

6. Lindroth JE, Schmidt JE, Carlson CR. A comparison between masticatory muscle pain patients and intracapsular pain patients on behavioral and psychosocial domains. J Orofac Pain. 2002;16:27783.

7. McNeill C. History and evolution of TMD concepts. Oral Surg Oral Med Oral Pathol Oral Radiol Endod. 1997;83:51-60.

8. Collesano V, Segù M, Masseroli C, Manni R. Temporomandibular disorders and sleep disorders: which relationship?. Minerva Stomatol. 2004;53:661-8.

9. Kuttila M, Niemi PM, Kuttila S, Alanen P, Le Bell Y. TMD treatment need in relation to age, gender, stress, and diagnostic subgroup. J Orofac Pain. 1998;12:67-74.

10. Galdon MJ, Durá E, Andreu Y, Ferrando M, Poveda R, Bagán JV. Multidimensional approach to the differences between muscular and articular temporomandibular patients: coping, distress, and pain characteristics. Oral Surg Oral Med Oral Pathol Oral Radiol Endod. 2006;102:40-6.

11. Sieber M, Grubenmann E, Ruggia GM, Palla S. Relation between stress and symptoms of craniomandibular disorders in adolescents. Schweiz Monatsschr Zahnmed. 2003;113:648-54.

12. Miyake R, Ohkubo R, Takehara J, Morita M. Oral parafunctions and association with symptoms of temporomandibular disorders in Japanese university students. J Oral Rehabil. 2004;31:518-23.

13. Elfving L, Helkimo M, Magnusson T. Prevalence of different temporomandibular joint sounds, with emphasis on disc-displacement, in patients with temporomandibular disorders and controls. Swed Dent J. 2002;26:9-19.

14. Gross A, Gale EN. A prevalence study of the clinical signs associated with mandibular dysfunction. J Am Dent Assoc. 1983;107:932-6.

15. Hirsch C, John MT, Lautenschläger C, List T. Mandibular jaw movement capacity in 10-17-yr-old children and adolescents: norma- 
tive values and the influence of gender, age, and temporomandibular disorders. Eur J Oral Sci. 2006;114:465-70.

16. Masumi S, Kim YJ, Clark GT. The value of maximum jaw motion measurements for distinguishing between common temporomandibular disorder subgroups. Oral Surg Oral Med Oral Pathol Oral Radiol Endod. 2002;93:552-9.

17. Pullinger AG, Seligman DA. Quantification and validation of predictive values of occlusal variables in temporomandibular disorders using a multifactorial analysis. J Prosthet Dent. 2000;83:66-75.

18. Kavuncu V, Sahin S, Kamanli A, Karan A, Aksoy C. The role of systemic hypermobility and condylar hypermobility in temporomandibular joint dysfunction syndrome. Rheumatol Int. 2006;26:25760.

19. Magnusson T, Karlsson C. Clinical impact of radiological examinations of patients with suspected temporomandibular disorders. Swed Dent J. 2002;26:67-74.

20. Crow HC, Parks E, Campbell JH, Stucki DS, Daggy J. The utility of panoramic radiography in temporomandibular joint assessment. Dentomaxillofac Radiol. 2005;34:91-5. 